International Journal of Trend in Scientific Research and Development (IJTSRD)

Volume: 3 | Issue: 3 | Mar-Apr 2019 Available Online: www.ijtsrd.com e-ISSN: 2456 - 6470

\title{
IOT Based Weather Monitoring and Reporting System Project
}

\author{
Anita M. Bhagat ${ }^{1}$, Ashwini G. Thakare1, Kajal A. Molke1, Neha S. Muneshwar ${ }^{1}$, Prof. V. Choudhary ${ }^{2}$ \\ ${ }^{1}$ BE Student, ${ }^{2}$ Associate Professor \\ 1,2Department of Electronics and Telecommunication, \\ 1,2J.D. College of Engineering and Management, Nagpur, Maharashtra, India
}

\begin{abstract}
How to cite this paper: Anita M. Bhagat | Ashwini G. Thakare | Kajal A. Molke | Neha S. Muneshwar | Prof. V.Choudhary "IOT Based Weather Monitoring and Reporting System Project" Published in International Journal of Trend in Scientific Research and Development (ijtsrd), ISSN: 24566470, Volume-3 | Issue-3, April 2019, pp.365-367, URL: http://www.ijtsrd.co $\mathrm{m} /$ papers/ijtsrd216 77.pdf

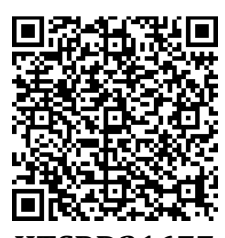
IITSRD21677
\end{abstract}

\section{ABSTRACT}

The IOT based Weather Monitoring and Reporting System project is used to get Live reporting of weather conditions. It will Monitor temperature, humidity, moisture and rain level. Suppose Scientists/nature analysts want to monitor changes in a particular environment like volcano or a rain-forest. And these people are from different places in the world. In this case, SMS based weather monitoring system has some limitations. Since it sends SMS to few numbers. And time for sending SMS increases as the number of mobile numbers increases. In order to know the information about weather of a perticular place then they have to visit that particular sites. Where everyone can see it.

Copyright (C) 2019 by author(s) and International Journal of Trend in Scientific Research and Development Journal. This is an Open Access article distributed under the terms of the Creative Commons

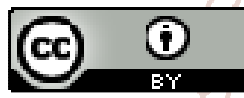
Attribution License (CC BY 4.0) (http://creativecommons.org/licenses/ by $/ 4.0$ )

\section{INTRODUCTION}

Climatic change and environmental monitoring have received much attention recently. Man wants to stay updated about the latest weather conditions of any place like a college campus or any other particular building. Since the world is changing so fast so there should be the weather stations. Here in this paper we present a weather station that is very helpful for any places. This weather station is based on IOT (internet of things). It is equipped with environmental sensors used for measurements at any particular place and report them in real time on cloud. To accomplish this we used Arduino Uno and different environmental sensors like DHT11, soil moisture sensor and rain drop sensor. The sensors constantly sense the weather parameters and keeps on transmitting it to the online web server over a wifi connection. The weather parameters are uploaded on the cloud and then provides the live reporting of weather information. This paper also focuses on the IOT application in the new generation of environmental information and provides a new paradigm for environmental monitoring in future. The system has been development particularly in the view of building smart city by giving the weather update of any particular place like a particular office or room.
Internet of Things (IOT):

It is the future technology of connecting the entire world at one place. All the objects, things and sensors can be connected to share the data obtained in various locations and process/analyses that data for coordinating the applications like traffic signaling, mobile health monitoring in medical applications and industrial safety ensuring methods, etc. As per the estimation of technological experts, 50 billion objects will be connected in IOT by 2020. IOT offers wide range of connectivity of devices with various protocols and various properties of applications for obtaining the complete machine to machine interaction.

\section{LITERATURE REVIEW:}

In today's world many pollution monitoring systems are designed by different environmental parameters. Existing system model is presented IOT based Weather monitoring and reporting system where you can collect, process, analyze, and present your measured data on web server. Wireless sensor network management model consists of end device, router, gateway node and management monitoring center. End device is responsible for collecting wireless sensor network data, and sending them to parent node, then data are sent to gateway node from parent node directly or by router. After receiving the data from wireless sensor network, gateway node extracts data after analyzing and packaging them into Ethernet format data, sends them to the server. Less formally, any device that runs server software 
could be considered a server as well. Servers are used to manage network resources. The services or information provided through the Internet that are connected through LAN and made available for users via smart phones, web browser or other web browser devices to make the system more intelligent, adaptable and efficient.

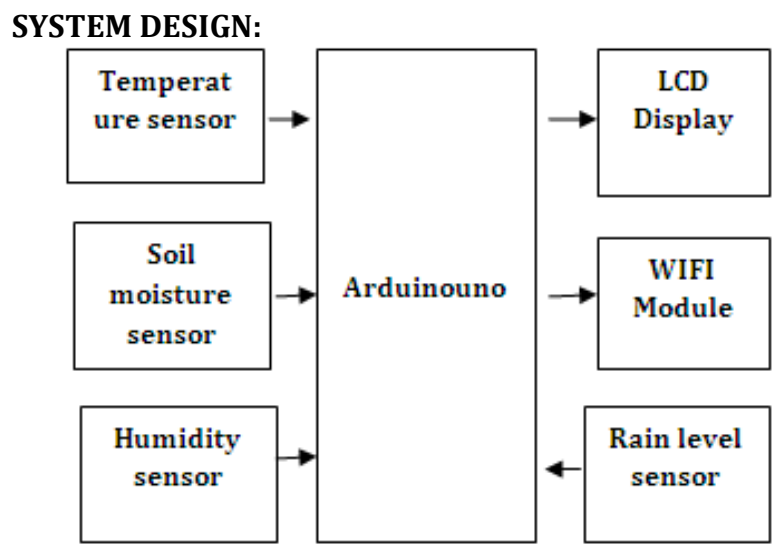

In IOT enabled weather monitoring system project, Arduino Uno measures four weather parameters using four respective sensors. These sensors are temperature sensor, humidity sensor, moisture sensor and rain level sensor. These four sensors are directly connected to Arduino Uno. Arduino Uno has inbuilt Analog to digital converter. Arduino calculates and displays these weather parameters on LCD display. Then it sends these parameters to Internet using IOT techniques. The process of sending data to the internet using $\mathrm{Wi}-\mathrm{Fi}$ is repeated after constant time intervals. Then the user needs to visit a particular website to view this weather data. The project connects and stores the data on a web server. Hence user gets Live reporting of weather conditions. Internet connectivity or Internet connection with $\mathrm{Wi}-\mathrm{Fi}$ is compulsory in this IOT based weather monitoring reporting system project.

\section{Arduino Uno:}

Arduino Uno is a microcontroller board developed by Arduino. Which is an open-source electronics platform mainly based on AVR microcontroller Atmega328. The current version of Arduino Uno comes with USB interface, 6 analog input pins, $14 \mathrm{I} / 0$ digital ports that are used to connect with external electronic circuits. Out of $14 \mathrm{I} / 0$ ports, 6 pins can be used for PWM output. It allows the designers to control and sense the external electronic devices in the real world. This board comes with all the features required to run the controller and can be directly connected to the computer through USB cable that is used to transfer the code to the controller using IDE software, mainly developed to program Arduino. Programming languages like $\mathrm{C}$ and $\mathrm{C}++$ are used in IDE. Apart from USB, battery or AC to DC adopter can also be used to power the board. Arduino Uno are the most official versions that come with Atmega328 8-bit AVR Atmel microcontroller where RAM memory is $32 \mathrm{~KB}$.

\section{Rain level sensor:}

The rain sensor module is an easy tool for rain detection. It can be used as a switch when raindrop falls through the raining board and also for measuring rainfall intensity. The analog output is used in detection of drops in the amount of rainfall. Connected to $5 \mathrm{~V}$ power supply, the LED will turn on when induction board has no rain drop, and DO output is high. When dropping a little amount water, DO output is low, the switch indicator will turn on. Brush off the water droplets, and where restored to the initial state, outputs high level. A rain sensor or rain switch is a switching device activated by rainfall.

\section{Temperature \& Humidity Sensor:}

This DHT11 Temperature and Humidity Sensor features digital signal output.It is integrated with a high-performance 8-bit microcontroller. Its technology ensures the high reliability and excellent long-term stability. It has excellent quality, fast response, anti-interference ability and high performance. Each DHT11 sensors features extremely accurate calibration of humidity calibration chamber. The calibration coefficients stored in the OTP program memory, internal sensors detect signals in the process, we should call these calibration coefficients. The single-wire serial interface system is integrated to become quick and easy. Small size, low power, signal transmission distance up to 20 meters, enabling a variety of applications and even the most demanding ones. The product is 4-pin single row pin package. Convenient connection, special packages can be provided according to users need.

\section{Soil Moisture Sensor:}

Soil moisture sensors measure the contents in soil. Since the direct gravimetric measurement of free soil moisture requires removing, drying, and weighting of a sample, soil moisture sensors measure the volumetric water content indirectly by using some other property of the soil, such as electrical resistance, dielectric constant, or interaction with neutrons, as a proxy for the moisture content.

The relation between the measured property and soil moisture must be calibrated and may vary depending on environmental factors such as soil type, temperature, or electric conductivity. Reflected microwave radiation is affected by the soil moisture and is used for remote sensing in hydrology and agriculture. Portable probe instruments can be used by farmers or gardeners.

\section{WiFi Module:}

The Arduino Uno WiFi is an Arduino Uno with an integrated WiFi module. The board is based on the ATmega328P with an ESP8266 WiFi Module integrated. The ESP8266 WiFi Module is a self contained SoC with integrated TCP/IP protocol stack that can give access to your WiFi network (or the device can act as an access point). One useful feature of Uno WiFi is support for OTA (over-the-air) programming, either for transfer of Arduino sketches or WiFi firmware.

\section{LCD Display:}

A Liquid Crystal Display commonly abbreviated as LCD is basically a display unit built using Liquid Crystal technology. When we build real life/real world electronics based projects, we need a medium/device to display output values and messages. The most basic form of electronic display available is 7 Segment displays - which has its own limitations. The next best available option is Liquid Crystal Displays which comes in different size specifications. Out of all available LCD modules in market, the most commonly used one is $16 \times 2$ LCD Module which can display 32 ASCII characters in 2 lines. To establish a good communication between human world and machine world, display units play an important role. And so they are an important part of embedded systems. Display units - big or small, work on the same basic principle. Besides complex display units like 
graphic displays and 3D display, one must know working with simple displays like $16 \times 1$ and $16 \times 2$ units. The $16 \times 1$ display unit will have 16 characters and are in one line. The 16x2 LCD will have 32 characters in total 16in 1st line and another 16 in 2 nd line. There are two control pins, this gives the flexibility. The contrast bit and READ/WRITE are not often used so they can be shorted to ground. This puts LCD in highest contrast and read mode. We just need to control ENABLE and RS pins to send characters and data accordingly.

\section{ADVANTAGES:}

$>$ IOT weather mentoring system project using Arduino Uno is fully automated.

$>$ It does not require any human attention.

$>$ We can get prior alert of weather conditions

$>$ The low cost and efforts are less in this system

$>$ Accuracy is high.

$>$ Self Protection

$>$ Smart way to monitor Environment

$>$ Efficient

\section{APPLICATIONS:}

$>$ The weather forecasting plays very important role in the field of agriculture.

$>$ It is also helpful at places like volcano and rain forests.

$>$ It is quite difficult for a human being to stay for longer time at such places.

\section{FUTURE SCOPE:}

$>$ One can implement a few more sensors and connect it to the satellite as a global feature of this system.

$>$ Adding more sensor to monitor other environmental parameters such as C02,Pressure and Oxigen Sensor

$>$ In aircraft, navigation and military there is a great scope of this real-time system.

$>$ It can also be implemented in hospitals or medical institutes for the research \& study in "Effect of Weather on Health and Diseases", hence to provide better precaution alerts.
CONCLUSION:

By keeping the weather station in the environment for monitoring enables self protection (i.e., smart environment) to the environment. To implement this need to use the sensor devices in the environment for collecting the data and analysis. By using sensor devices in the environment, we can bring the environment into real life. Then the collected data and analysis results will be available to the user through the Wi-Fi. The smart way to monitor environment an efficient, low cost embedded system is presented in this paper. It also sent the sensor parameters to the cloud. This data will be helpful for future analysis and it can be easily shared to other users also. This model can be expanded to monitor the developing cities and industrial zones for pollution monitoring. To protect the public health from pollution, this model provides an efficient and low cost solution for continuous monitoring of environment.

\section{REFERENCES:}

[1] International Journal of Advanced Research in Computer and Communication Engineering ISO 3297:2007 Certified Vol. 5, Issue 9, September 2016

[2] International Journal of Engineering Trends and Technology (IJETT) - Volume 32 Number 2- February 2016

[3] International Journal of Engineering Science and Computing, May 2017

[4] Sagar J. S. T. , M. S. Balamurugan and J. A. Vivek, "A wireless framework for automotive monitoring systems," in Indian Journal of Science and Technology, Vol 8(19), IPL0146, August 2015

[5] https://www.openhacks.com/uploadsproductos/rain_s ensor_module.pdf

[6] http://designinformaticslab.github.io/productdesign_tu torial/2017/01/24/soilmoisture_sensor.html

[7] content://com.sec.android.app.sbrowser/readinglist/07 21190655.mhtml

[8] https://www.hackster.io/techmirtz/using-16x2-lcdwith-arduino-d89028

[9] https://www.arduino.cc/en/Guide/ArduinoUnoWiFi 